\title{
Reversing Synapse Loss in Alzheimer's Disease: Rho-Guanosine Triphosphatases and Insights from Other Brain Disorders
}

\author{
Roger Lefort
}

Published online: 15 January 2015

(C) The American Society for Experimental NeuroTherapeutics, Inc. 2015

\begin{abstract}
Alzheimer's disease (AD) is a monumental public health crisis with no effective cure or treatment. To date, therapeutic strategies have focused almost exclusively on upstream signaling events in the disease, namely on $\beta$-amyloid and amyloid precursor protein processing, and have, unfortunately, yielded few, if any, promising results. An alternative approach may be to target signaling events downstream of $\beta$ amyloid and even tau. However, with so many pathways already linked to the disease, understanding which ones are "drivers" versus "passengers" in the pathogenesis of the disease remains a tremendous challenge. Given the critical roles of Rho-guanosine triphosphatases (GTPases) in regulating the actin cytoskeleton and spine dynamics, and the strong association between spine abnormalities and cognition, it is not surprising that mutations in a number of genes involved in RhoGTPase signaling have been implicated in several brain disorders, including schizophrenia and autism. And now, there is mounting literature implicating Rho-GTPase signaling in AD pathogenesis as well. Here, I review this evidence, with a particular emphasis on the regulators of Rho-GTPase signaling, namely guanine nucleotide exchange factors and GTPaseactivating proteins. Several of these have been linked to various aspects of $\mathrm{AD}$, and each offers a novel potential therapeutic target for $\mathrm{AD}$.
\end{abstract}

Key Words Alzheimer's disease · Rho-GTPase · Eph · GEF · GAP · therapeutics

\section{R. Lefort $(\bowtie)$}

The Taub Institute for Research on Alzheimer's Disease and the Aging Brain, and Department of Pathology and Cell Biology, Columbia University Medical Center, New York, NY 10032, USA e-mail: r12040@cumc.columbia.edu

\section{Introduction}

In the mammalian nervous system, one of the most morphologically plastic structures in neuronal networks can be found along dendritic shafts, in the form of small protrusions called dendritic spines. During development and throughout adulthood, their numbers and morphology constantly evolve, reflecting changes in synapse formation and function, allowing for remodeling of neuronal circuits. It is generally believed that this inherent plasticity is the basis of learning and memory processes, driven by changes in signaling pathways and cytoskeletal protein rearrangement [1-5].

Given their importance in learning and memory, it is not surprising that dementia could stem from the disruption of normal dendritic spine function, an idea that dates back to well over 100 years ago, to Ramón y Cajal, who first postulated that "dementia could result when synapses between neurons are weakened as a result of a more or less pathological condition, that is, when processes atrophy and no longer form contacts, when cortical mnemonics or association areas suffer partial disorganization". Indeed, in the last few decades, multiple lines of evidence have shown that disruptions in dendritic spine shape, size, or number invariably accompany various brains disorders, such as schizophrenia [6-9], Angelman syndrome [10], and Rett syndrome [11-13], and may be even be a characteristic feature of traumatic brain injuries $[14,15]$. What do these various neuropsychiatric and neurodevelopmental diseases share in common? They all involve deficits in information processing and share a key common pathogenic substrate, namely dendritic spines. Alzheimer's disease (AD), however, represents an interesting case.

\section{$\mathrm{AD}$ is a Disease of Synapses}

While classified as a neurodegenerative disease, in the earliest clinical phases, patients with $\mathrm{AD}$, who otherwise exhibit 
normal sensory and motor functions, typically present with a remarkably pure impairment of cognitive abilities and amnestic symptoms. Mounting evidence suggests that these are due to prominent synapse loss, particularly in the hippocampus and throughout the cortex [16-20], the principal areas affected in $\mathrm{AD}$, and not to the loss of whole neurons. In fact, dendrite and synapse loss show a much stronger correlation with the associated cognitive decline than do neurofibrillary tangles or frank neuronal degeneration [17]. Additionally, the loss of synapses is often significantly more pronounced than would otherwise be predicted from simple neuronal loss, firmly placing synaptic dysfunction as a driver of AD-related cognitive decline rather than a byproduct [16-18]. At its core, AD is a disease of synapses. Consequently, correcting this loss of synapses represents a viable and arguably requisite therapeutic strategy, especially in the early stages of the disease, prior to massive neuronal loss.

\section{Rho-Guanosine Triphosphatases at the Center of AD Pathology}

The precise molecular nature of synapse loss in AD is not fully understood. However, previous studies in developing brains and in maturing neurons in culture provide valuable clues. Of particular interest is the Rho-family of guanosine triphosphatases (GTPases), a subfamily of the Ras superfamily of GTPases, which stimulate a wide array of cellular processes, including morphogenesis, cell migration, mitosis, and adhesion [21-23]. The Rho subfamily is further divided into 7 subfamilies (Rho, Rac, Cdc42, Rnd, RhoD, RhoBTB, and $\mathrm{RhoH}$ ), of which the most extensively studied members are RhoA, Rac1, and Cdc42 for their role in regulating the actin cytoskeleton, the main structural component of dendritic spines, and thus in controlling spine dynamics [24-27]. Because of their important roles in various cellular processes, their activities are tightly spatiotemporally regulated to ensure homeostasis.
There are 3 main modes of regulation of Rho-GTPase activity (Fig. 1) [28]. They are activated by guanine nucleotide exchange factors (GEFs), which catalyze the exchange of bound guanosine-5'-diphosphate for guanosine-5' -triphosphate, and inhibited by GTPase-activating proteins (GAP), which catalyze the hydrolysis of guanosine-5'-triphosphate to guanosine-5'-diphosphate. A third critical level of regulation is provided by the Rho-specific guanine nucleotide dissociation inhibitor family of proteins, which act to sequester the inactive pool of Rho-GTPases in the cytosol and prevent their interactions with GEFs at the cell membrane [29].

Aberrant Rho-GTPase signaling leads to widespread neuronal network dysfunction and eventual diseases, and has been proposed to cause certain diseases, including AD. Recent immunohistological studies suggest that the subcellular localization of RhoA may be altered in AD brains, with decreased staining in the neuropil and a marked increase in neurons, co-localizing with hyperphosphorylated tau inclusions [30]. Localization of Rac1 and Cdc42 were reported to be unchanged. RhoA mislocalization can also be seen in the human amyloid precursor protein (hAPP) Tg2576 (Swedish mutation) AD mouse model, which shows decreased levels in synapses but increased levels in dystrophic neurites [30]. At 12-18 months of age, these mice have also been reported to have significantly increased RhoA levels and decreased Rac1 levels in the brain [31]. Our recent studies in the hAPP J20 (Swedish and Indiana mutations) AD mouse model show a strong correlation between dendritic spine loss and behavioral deficits with a significant increase in RhoA activity [32]. In vitro studies in cultured cells also reflect these changes. $A \beta$ oligomers trigger a significant increase in RhoA activity in both SY5Y cells and in cultured hippocampal neurons [31-34]. Cdc42 and Rac1 levels have been reported to be elevated in select neuronal populations in AD brains compared with age-matched controls and have been proposed to perhaps play a role in activating cell cycle-related genes [35]. Interestingly, in these studies $\mathrm{Cdc} 42$ and Rac1 show considerable overlap with early cytoskeletal abnormalities

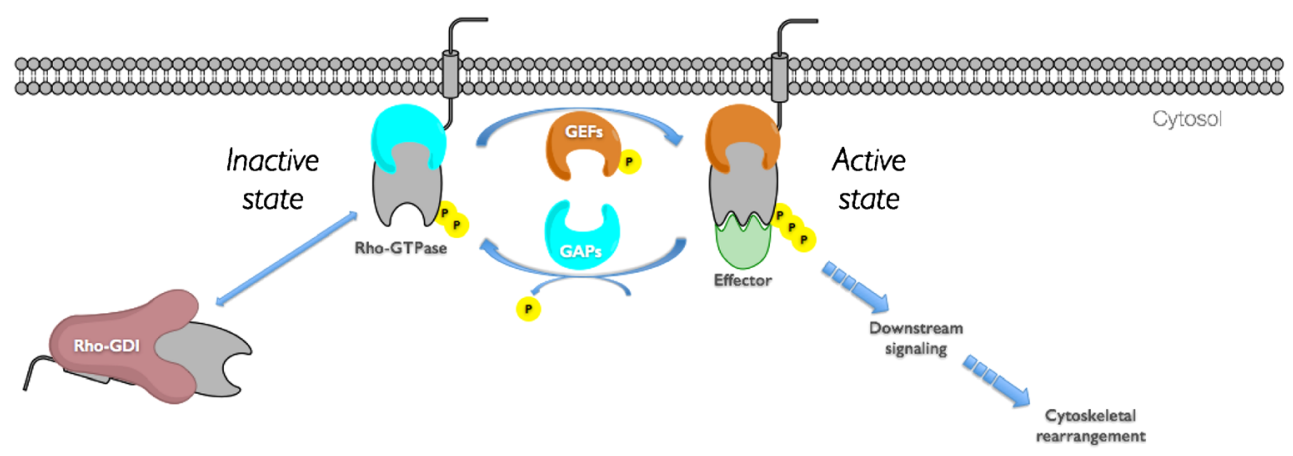

Fig. 1 Regulation of Rho-guanosine triphosphatase (GTPase) activity. Rho-GTPases are activated by guanine nucleotide exchange factors (GEFs), which catalyze the exchange of bound guanosine-5'diphosphate (GDP) for guanosine-5'-triphosphate (GTP), and inhibited

by GTPase activating proteins (GAPs), which catalyze the hydrolysis of GTP to GDP. A third level of regulation is through binding of Rho GDPdissociation inhibitor (Rho-GDI), which inhibits nucleotide exchange and membrane association. $\mathrm{P}=$ phosphorylation 
suggesting that these changes are an extremely proximal event in the pathogenesis of the disease. These findings are consistent with studies showing increased $\mathrm{Cdc} 42$ and Rac1 activity in hippocampal neurons treated with fibrillar $\beta$-amyloid $(A \beta)$ [36]. Intriguingly, both RhoA and Rac1 have been implicated in the processing of APP and A $\beta 42$ production. It has been proposed that Rac1 activity might promote $A \beta 42$ production by positively regulating APP gene synthesis [37], and/or by shifting the substrate specificity of $\gamma$-secretase between Notch1 and APP [38]. Moreover, cleavage of APP may be, at least in part, dependent on RhoA signaling, as $A \beta 42$ production is significantly reduced by treatment with nonsteroidal anti-inflammatory drugs that block RhoA activity or that of the RhoA effector ROCKII in vivo in a transgenic AD mouse model $[39,40]$.

Taken together, the evidence suggests that Rho-GTPases play a fundamental role in several aspects of $\mathrm{AD}$ pathology, from APP processing and $A \beta 42$ production to the widespread synaptic dysfunction associated with the disease. While the interplay between the various signaling pathways remains unclear, RhoGTPases may provide attractive therapeutic targets for AD.

\section{Direct Modulation of Rho-GTPases for AD Treatment}

The idea that the direct pharmacological modulation of RhoGTPase activity might restore normal synaptic function and cognition in AD has some precedence. Previous studies have shown that cytotoxic necrotizing factor 1 (CNF1), a bacterial protein produced by Escherichia coli that activates RhoA, Rac1, and Cdc42, can promote the formation of spine-like structures in cultured neurons. Local injection of CNF1 has been shown to increase dendritic spine density in the rat visual cortex [41]. More importantly, these newly formed dendritic spines appear to be functional, as intracerebroventricular (ICV) injection of CNF1 can persistently (up to 90 days post-treatment) increase hippocampal neurotransmission, long-term potentiation (LTP), and memory in wild-type mice [42-44]. These benefits may also translate to other neurological disorders. For example, ICV injection of CNF1 has been reported to reduce locomotor hyperactivity and to partially correct memory deficits typically seen in the hAPP (Swedish and Indiana mutations) TgCRND8 AD mouse model [45]. CNF1 can also improve the behavioral and astrocytic deficits in a mouse model of Rett syndrome [46]. It should be pointed out that as CNF1 targets all 3 Rho-GTPases (i.e., RhoA, Rac1, and Cdc42) for activation, it is unlikely that it simply corrects a possible imbalance in Rho-GTPase signaling in $\mathrm{AD}$, and the possibility that the beneficial effects could be nonspecific and unrelated to any direct action on $\mathrm{AD}$ pathology cannot be ruled out. To date, selective modulation of individual RhoGTPases has not been evaluated in the context of AD treatment, but the involvement of Rho-GTPases in several cancers has driven considerable pharmaceutical research, and several specific inhibitors have been developed for cancer-related therapies [47]. Whether or not any of these specific inhibitor/modulators can be beneficial for patients with $\mathrm{AD}$ remains to be seen, but they certainly represent a worthwhile area for investigation. One important caveat to note, however, is that total inhibition of Rho-GTPases might, in fact, not be an optimal strategy. Rho-GTPases play important physiological roles in normal synaptic functions, and their activities are essential for learning and memory, and for the establishment of LTP [48]. As a result, total inhibition may interfere with longterm signaling required for spine structural plasticity [49]. An alternative approach may be to manipulate selectively the activity of the regulators of Rho-GTPase signaling.

\section{Aberrant Regulation of Rho-GTPase Signaling in AD}

While aberrant Rho-GTPase signaling may be associated with several neurological diseases, to date, none of them, with the exception of certain cancers [50], can be directly attributable to loss- or gain-of-function mutations in the protein themselves. Unlike Ras proteins, which are frequently mutated, especially in cancer $(\sim 30 \%)$, activity-altering mutations in the Rho proteins themselves are extremely rare. Rather, the aberrant signaling appears to stem from the overexpression, mutation, or dysregulation of proteins that control RhoGTPase activity, such as overactivation or overexpression of GEFs and inactivation of GAPs or guanine nucleotide dissociation inhibitors [51, 52].

One of the first such described examples is in the wellknown neurodevelopmental disease, $\mathrm{X}$-linked mental retardation, characterized by a severe deficit in cognitive functions, with the absence of any gross abnormalities in brain structures [53]. However, ultrastructural analysis suggests that this may be caused by severe defects in morphology of dendritic spines, resulting in altered neuronal network formation [54]. This is thought be caused, at least in part, to a loss of function of oligophrenin-1, a RhoGAP expressed in both fetal and adult brains $[55,56]$, which stimulates the GTPase activities of RhoA, Rac1, and Cdc42 [57], although more recent data suggest that its activity may be specific for RhoA [58]. A loss of function of oligophrenin-1 leads to increased RhoA activity, eventually culminating in abnormal dendritic spine morphology. Mutations in ARHGEF6, which encodes a Rac1/Cdc42 GEF, known as $\alpha$ PIX/Cool-2, has also been described in patients with X-linked mental retardation [59], possibly resulting in decreased Rac1/serine/threonine-protein kinase 3 (PAK3) signaling [60]. Oligophrenin-1 has not been directly associated with AD. However, recent in vitro data have identified it as a potential binding partner for intersectin-1 [61], a multifunctional adaptor protein involved in endocytosis and exocytosis, which has itself been associated with $\mathrm{AD}$ and Down syndrome 
[62]. While the involvement of oligophrenin-1 in AD remains speculative, several Rho-GTPase regulatory proteins have been linked to $\mathrm{AD}$ (Table 1).

\section{Kalirin-7}

Kalirin was originally discovered in 1996 as a novel protein that interacts with the cystolic C-terminus of peptidylglycine $\alpha$ amidating monooxygenase, an integral membrane peptideprocessing enzyme [63]. Kalirin has since emerged as a major Rac1-GEF and as a key regulator of structural and functional plasticity at dendritic spines [64-68]. Several isoforms of the kalirin gene (KALRN), stemming from various promoters and transcriptional starts, have been discovered over the years, including the major isoforms kalirin-5 kalirin-7, kalirin-9, kalirin12, and Duet [69-71]. Several of these isoforms have been linked to a wide range of human diseases, including stroke and addiction [72], but kalirin-7 has been of particular interest because of its possible involvement in neuropsychiatric diseases.

Kalirin-7 (Duo in humans) is the most abundant kalirin isoform in the adult brain, where its expression is largely limited to central nervous system neurons, localizing specifically at the postsynaptic end of excitatory synapses. Interestingly, in rodents, kalirin-7 protein levels are almost undetectable at birth, only increasing at postnatal day 14 , which coincides with the onset of synaptogenesis [73, 74]. Kalirin-7 plays a key role in the regulation of the size and density of dendritic spines, where it interacts with several PDZ domain-containing proteins [64-68].

Intriguingly, several recent studies have shown kalirin-7 mRNA and protein levels to be decreased in AD hippocampal tissue [75-77]. The functional consequences of this decrease are not clear, but, interestingly, kalirin-7 associates with inducible nitric oxide synthase (iNOS) in the hippocampus resulting in the downregulation of its enzymatic activity $[76,78]$. These data are in line with the findings that iNOS levels and activity are increased in $\mathrm{AD}[79,80]$, suggesting that the decrease of kalirin-7 in patients with $\mathrm{AD}$ contributes to further augment iNOS activity and exacerbate AD pathology [76]. Kalirin-7 decrease may also have a critical causative role in dendritic spine pathology in AD. Kalirin-7 knockout (KO) mice show a significant decrease in hippocampal dendritic spine density, deficits in LTP, and impaired cognitive function [64]. These synaptic deficits are thought to be due to abnormally attenuated signaling by Rac1, the main target of Kalirin-7 [65-67].

Much work remains to be done to fully dissect the role of kalirin-7 in AD pathogenesis, especially in mediating dendritic spine pathology. However, the available evidence is compelling. Restoring the depleted levels of kalirin-7 mRNA, or altering its protein function or that of its downstream targets may be a viable therapeutic strategy to rescue $\mathrm{AD}$-associated synaptic dysfunction and dendritic spine loss. The recent discoveries that kalirin-7 may also be implicated in schizophrenia, where its levels are also reduced, are of particular interest and suggest perhaps a common mechanism between these two diseases. Therapeutic strategies targeting kalirin-7 may also prove beneficial for $\mathrm{AD}$ treatment.

\section{Dedicator of Cytokinesis 3}

Dedicator of cytokinesis (Dock) proteins are members of a family of GEF proteins (A, B, C, D) that differ from other GEFs in that they lack 2 common motifs, namely the double homology (DH) domain and the pleckstrin homology (PH)

Table 1 Involvement of Rho-guanosine triphosphatases (GTPases) and their regulators in the pathogenesis of Alzheimer's disease (AD)

\begin{tabular}{lllll}
\hline & Name & Change in AD & Regulators/target(s)/effector(s) & Proposed roles in AD [reference(s)] \\
\hline Rho-GTPase & RhoA & $\uparrow$ & ROCKI, ROCKII & $\begin{array}{c}\text { Synaptic dysfunction; dystrophic neunites; dendritic spine loss; } \\
\text { A } \beta 42 \text { production [30-34, 39, 40] } \\
\text { Cytoskeletal abnormalities; altered APP processing; increased } \\
\end{array}$ \\
& Rac1 & $\uparrow \downarrow$ & PAK1, PAK3 & APP synthesis [31, 35-38] \\
& Cdc42 & $\uparrow \downarrow$ & PAK2, PAK3 & Cytoskeletal abnormalities; cell cycle re-entry [31, 35, 36] \\
GEFs/GAPs & Dock3 & $\downarrow$ & Rac1 & Tau hyperphosphorylation and neurofibrillary tangles; APP \\
& & & degradation; A $\beta$ production [85, 86]
\end{tabular}

$\uparrow=$ upregulated activity or protein expression; $\downarrow=$ repressed activity or protein expression; GEF $=$ guanine nucleotide exchange factor; GAP $=$ GTPaseactivating protein; EphR = erythropoietin-producing hepatocellular receptor; ND = not determined; ROCK $=$ rho-associated, coiled coil-containing protein kinase; $\mathrm{PAK}=$ serine/threonine-protein kinase; $\mathrm{APP}=$ amyloid precursor protein; $\mathrm{A} \beta=\beta$-amyloid; NMDA $=N$-methyl-D-aspartate 
domain [81]. Instead, they contain two Dock homology regions (DHRs), DHR-1 and DHR-2, which play important roles for the membrane targeting and GEF activity of Docks, respectively. So far, 11 Dock proteins have been identified in the mammalian system (Docks 1-11), playing important physiological functions in diverse brain development processes [82]. Several Dock proteins have already been linked to various neuropsychiatric and neurodegenerative diseases, including autism spectrum disorder, schizophrenia, and Parkinson's disease.

Dock3 (also known as modifier of cell adhesion), a member of the DOCK-B subfamily, was originally discovered as a presenilin-1 (PS1)-interacting protein, highly expressed in the cortex and hippocampus, which hinted at a possible role in $\mathrm{AD}$ pathogenesis [83]. Indeed, analysis of postmortem brain tissue patients revealed a significant decrease in total Dock3 protein in AD brains. As Dock3 is a Rac1-specific GEF [84], its depletion is consistent with reduced Rac1 activity in $\mathrm{AD}$ neurons. Interestingly, overexpression of Dock 3 stimulates the phosphorylation of tau protein and has been found to localize to the insoluble fraction of extracts from $\mathrm{AD}$ brains, where it associates with neurofibrillary tangles [85]. Dock3 is also thought to play a role in the regulation of APP processing. Expression of Dock3 promotes the ubiquitin-proteasome system-mediated degradation of APP and significantly reduces $A \beta$ secretion [86]. Whether decreased Dock3 levels in AD contribute to increased $A \beta$ levels is unknown, but it further emphasizes the importance of dysregulated Rho-GTPase regulators in $\mathrm{AD}$.

\section{Erythropoietin-Producing Hepatocellular Receptor Signaling}

Another class of molecules that has garnered a great deal of attention in the past few years is the erythropoietin-producing hepatocellular (Eph) family of receptor tyrosine kinases. Already implicated in various physiological and pathological processes in many cell types and organs [87], Eph receptors (EphRs) have emerged as key players in neuropsychiatric diseases [88]. EphRs bind to membrane-tethered ephrin receptorinteracting proteins known as ephrin ligands, located on adjacent cells, to initiate a bidirectional signaling cascade downstream of both the receptor (forward signaling) and the ligand (reverse signaling) [89]. EphRs are subdivided into 2 classes, based on sequence similarities and the preferred binding ligand: EphA and EphB. Each subclass of EphR is comprised of several members (EphA1-A8 and EphB1-B6). As a general rule, EphA receptors usually bind to glycosylphosphatidylinositol anchor-linked ephrin A ligands, while EphB receptors tend to bind transmembrane ephrin B ligands; however, EphR/ephrin cross-reactivity does occur [87].

One of the key functions of EphR is to regulate cytoskeletal dynamics through Rho-GTPases, more specifically through RhoA. EphR kinase activation can activate RhoA directly, or indirectly through the EphR-interacting nucleotide exchange factor, Ephexin, which can activate RhoA. The ability to modulate Rho-GTPase activity in neurons is critical during development to regulate neurite outgrowth, growth cone collapse, and axon guidance [87], and, more recently, as regulators of excitatory synapse formation, function, and plasticity in the central nervous system [88]. Indeed, several EphR and ligands localize with postsynaptic density marker PSD95 at excitatory synapses in hippocampal neurons, including EphA4, EphB1, EphB2, and EphB3 [90-95]. To date, at least 2 EphRs have been linked to $\mathrm{AD}$.

\section{EphB2/Ephexin-5}

Considerable progress has been made in characterizing the mechanisms by which EphB receptors regulate excitatory synaptic function and synapse development. Targeted deletion of $\mathrm{EphB} 2$ in rodents has revealed that EphB2 plays a critical role in regulating $\mathrm{N}$-methyl-D-aspartate-receptor calcium signaling and in the establishment of LTP, particularly in the hippocampal perforant path [96-98]. More recently, compelling evidence has shown EphB2 to be directly implicated in dendritic spine formation during development through the temporally precise inhibition of RhoA signaling [99]. One of the key components of this pathway is ephexin- 5 ( 1 of 5 closely related GEFs), which directly interacts with EphB2. Activation of EphB2 signaling leads to the tyrosine phosphorylation and degradation of ephexin-5, resulting in suppressed RhoA activation in neurons, thereby allowing for synapse development [99]. Consistent with these findings, EphB1/EphB2/EphB3 triple $\mathrm{KO}$ mice have fewer mature cortical and hippocampal excitatory synapses, suggesting that any depletion of EphB levels in the brain could negatively affect synaptic structures.

Indeed, a dramatic decrease of EphB2 receptor levels has been reported in postmortem brain tissue from patients with AD with mild cognitive deficits $[100,101]$. Similarly, a decrease in EphB2 is also observable in the hAPP transgenic AD mouse model as early as 2 months of age, prior to onset of behavioral deficits. This strongly suggests that a decrease in EphB2 may be a causative factor rather than a simple consequence of synaptic dysfunction in AD. While EphB2 can be processed by $\gamma$-secretase-mediated cleavage [102], depletion of EphB2 levels appears to stem from increased internalization and degradation of the receptor rather than increased processing, explaining the decrease in both cell surface and total EphB2 levels in AD neurons [101]. More importantly, the evidence suggests that this abnormal process may be triggered by $\mathrm{A} \beta$ oligomers, which exhibit a high affinity for the extracellular domain of EphB2, directing the receptor to the ubiquitin-proteasome system for degradation [101]. Loss of normal EphB2 function results in its inability to target ephexin-5 for degradation, resulting in hyperactivation of RhoA in neurons, which may account for the loss of dendritic 
spines and synaptic proteins, such as N-methyl-D-aspartate receptors. Intriguingly, EphB2 can also associate with intersectin (Cdc42 GEF) and kalirin (Rac1 GEF), both of which are implicated in AD (discussed above), which cocluster with activated EphB2 at synaptic sites to regulate EphB2-mediated morphogenesis and maturation of dendritic spines in cultured neurons [103, 104]. Whether EphB2 depletion in $\mathrm{AD}$ is directly related to reduced kalirin-7 or intersectin-1 levels is unknown.

One of the more intriguing findings from the study by Cissé et al. [101] was that reversing EphB2 depletion in a subset of granule neurons in the dentate gyrus of hAPP mice was sufficient to counteract the synaptotoxic effects of $A \beta$ : dentate gyrus LTP, and learning and memory, were completely restored to normal levels in these mice. Again, this points to the EphB2 receptor as a possible intervention point for $\mathrm{AD}$ treatment. However, as the authors pointed out, whether increasing EphB2 levels in other brain regions will be as welltolerated as in the dentate gyrus remains unknown at this point.

\section{EphA4/Ephexin-1}

Another key EphR involved in synaptic function and dendritic spine morphology is EphA4. However, contrary to the mechanism of EphB2 receptors, ephrin ligand-mediated activation of EphA4 results in the reduction of synaptic proteins, such as GluR1 and PSD95, and in the retraction and elimination of dendritic spine at excitatory synapses $[91,92,105]$. Of note, however, EphA4 KO mice also exhibit significant defects in dendritic spines [92]. While the overall dendritic arborization of pyramidal neurons remains normal in these mice, the dendritic spines appear significantly longer and have irregular shapes and organizations. At least 2 mechanisms have been described for how EphA4 regulates spine dynamics in neurons. The first involves glial ephrin-A3 binding to neuronexpressed EphA4 [92, 106]. Binding of astrocyte-expressed ephrin-A3 to postsynaptic EphA4 activates forward signaling in neurons, resulting in dendritic spine retraction, while simultaneously triggering reverse signaling in astrocytes to downregulate glutamate transport. The downstream components of these pathways have yet to be elucidated, but the other described mechanism does offer some clues.

Binding of EphA4 to its highest affinity ligand [107], ephrin-A1 also results in dendritic spine retraction [91]. This interaction results in the recruitment of a complex involving Cdk5 (implicated in AD) and $\mathrm{p} 35$, resulting in the activation of another important RhoA-GEF, ephexin-1. Activation of EphA4 by ephrin-A1 thus triggers activation of RhoA and the collapses of dendritic spines. Could increased RhoA in $\mathrm{AD}$ be the result of aberrant EphA4 signaling in neurons?

There have been conflicting reports on the levels of EphA4 in AD. While one study has reported a significant decrease in
EphA4 protein levels in AD brain tissue from patients with mild cognitive deficits and in the Tg2576 AD mouse model [100], analysis of synaptosomes from patients with AD reveals a significant increase in EphA4 mRNA levels [108]. Another study has shown that the levels of EphA4 are not changed in $\mathrm{AD}$, but immunohistological localization of EphA4 in early stage AD (Braak stage II) reveals an altered distribution in AD brains, with high immunoreactivity in plaque-like structures, especially in the vicinity of neuritic plaques [109]. Interestingly, A $\beta$ oligomers have also been shown to trigger a redistribution of EphA4 from dendritic spines to the dendritic shaft of hippocampal neurons, preceded by a significant increase in EphA4 receptor activation [110]. A similar activation of EphA4 receptor is also observed in hAPP/PS1 transgenic AD mice, with no change in total protein levels, seemingly at odds with the decrease reported in Tg2576 AD mice [100]. These discrepancies may be due to differences between the AD mouse models (hAPP vs hAPP/PS1), or simply to the age at which these analyses were performed. Aberrant Rac1 activity in AD could also be caused by abnormal EphA4 signaling. Indeed, EphA4 has been identified as a substrate for both $\beta$-secretase (BACE1) and $\gamma$ secretase $[111,112]$. EphA4 processing by $\gamma$-secretase upon increased synaptic activity generates EphA4 intracellular domain fragments, which trigger the formation of dendritic spines through a Rac1-dependent pathway. More importantly, AD-linked PS1 mutations (PS1M146L and PS1E280A) can impair EphA4 processing, resulting in reduced levels of EphA4 intracellular domain fragments and decrease Rac1/ PAK1 signaling [112]. The exact GEF/GAP involved is so far unknown, but the Rac1 GAP $\alpha 2$-chimaerin, which interacts with EphA4, may be a good candidate for future research [113]. Taken together, the evidence suggests that EphA4 may be a critical regulator of dendritic spine morphogenesis, able to regulate both RhoA and Racl activities. Thus, aberrant EphA4 signaling, either through increased expression and/or decreased $\gamma$-secretase processing, could account for an imbalance in signaling between RhoA and Rac1, similar to that described in AD. Could restoring normal EphA4 signaling then be a viable therapeutic strategy for AD? Two recent studies suggest that this may be the case.

In vitro use of the peptide-based inhibitor of EphA4 signaling KYL [114] was shown to block $A \beta$-induced synaptic dysfunction, reversing both the loss of dendritic spines in cultured hippocampal neurons and the blockage of LTP induction in hippocampal slices by A $\beta$ oligomers $[110,115]$. Moreover, ICV injections ( $\sim 3$ weeks) of KYL was shown to be effective in blocking EphA4 signaling in the hippocampus of APP/PS1 transgenic $\mathrm{AD}$ mice, resulting in the complete rescue of impaired LTP normally observed in these mice [115]. Finally, molecular docking studies have led to the identification of the small chemical, rhynchophylline, as a potent EphA4 signaling inhibitor, which was shown to completely 
block EphA4 activity in the hippocampus when administered orally ( $\sim 3-4$ weeks) to APP/PS-1 and Tg2576 AD mice [115]. More importantly, this treatment was able to completely restore normal synaptic plasticity in these mice, making a strong case for an EphA4-based therapeutic strategy for AD.

\section{Perspectives: A Wealth of Possible New AD Targets}

As I have discussed throughout this review, the evidence for the involvement of Rho-GTPases in the pathogenesis of $\mathrm{AD}$ is mounting. Several aspects of the disease, from synaptotoxicity to APP processing, and $\mathrm{A} \beta$ production to tau pathology, elicit the participation of at least one RhoGTPase and/or one of their regulators. And while the precise contribution of each signaling pathway remains unclear, significant progress continues to be made. Each one represents a novel potential therapeutic target for $\mathrm{AD}$, addressing not only the main symptom of the disease, that is cognitive dysfunction, but perhaps the primary drivers of the disease, namely increased $A \beta$ and tau. This is an important consideration as we do not know whether addressing the symptom alone can alter disease progression. The critical point to take away is that this aberrant signaling appears to cause an imbalance in RhoGTPase signaling, whereby RhoA is overly activated and Rac1 activity is attenuated. Restoring this balance could be a key step in preventing the loss of synapses associated with the disease.

The hunt for small molecules or peptides capable of accomplishing this remains a challenging task. However, as this imbalance is commonly found in other neuropsychiatric diseases, therapeutic strategies for other diseases may also prove beneficial for AD. Once example may be for Angelman syndrome, where the loss of function of the E3 ligase Ube3A leads to increased RhoA activity and the abnormal pruning of dendritic spines. One current strategy being explored for Angelman syndrome is to restore normal Ube3A expression in order to decrease RhoA activity. A similar strategy for AD may very well work to restore normal RhoA signaling and rescue the loss of synapses. Another interesting area for inspiration may be in cancer research, where RhoGTPase dysfunction is well documented [116]. Small molecules are constantly being evaluated for the treatment of several cancers and it would certainly be worthwhile to evaluate them for AD in future studies. Ultimately, targeting the pathogenic pathways may open new avenues to combination therapies, including those designed to lower $\mathrm{A} \beta$ or Tau levels in the brain. As biomarker profiling improves, these could be customized to individuals as they progress through various pathological stages of the disease. This may prove to be the best strategy to intervene and cure AD.
Required Author Forms Disclosure forms provided by the authors are available with the online version of this article.

\section{References}

1. Greenough WT, West RW, DeVoogd TJ. Subsynaptic plate perforations: changes with age and experience in the rat. Science. 1978;202(4372):1096-1098.

2. Pietropaolo S, Feldon J, Alleva E, Cirulli F, Yee BK. The role of voluntary exercise in enriched rearing: a behavioral analysis. Behavioral neuroscience. 2006;120(4):787-803.

3. Leuner B, Falduto J, Shors TJ. Associative memory formation increases the observation of dendritic spines in the hippocampus. The Journal of neuroscience: the official journal of the Society for Neuroscience. 2003;23(2):659-665.

4. Leuner B, Shors TJ. New spines, new memories. Molecular neurobiology. 2004;29(2):117-130.

5. Bailey CH, Kandel ER. Structural changes accompanying memory storage. Annual review of physiology. 1993;55:397-426.

6. Garey LJ, Ong WY, Patel TS, et al. Reduced dendritic spine density on cerebral cortical pyramidal neurons in schizophrenia. Journal of neurology, neurosurgery, and psychiatry. 1998;65(4):446-453.

7. Glantz LA, Lewis DA. Decreased dendritic spine density on prefrontal cortical pyramidal neurons in schizophrenia. Archives of general psychiatry. 2000;57(1):65-73.

8. Sweet RA, Henteleff RA, Zhang W, Sampson AR, Lewis DA. Reduced dendritic spine density in auditory cortex of subjects with schizophrenia. Neuropsychopharmacology: official publication of the American College of Neuropsychopharmacology. 2009;34(2): 374-389.

9. Kolomeets NS, Orlovskaya DD, Rachmanova VI, Uranova NA. Ultrastructural alterations in hippocampal mossy fiber synapses in schizophrenia: a postmortem morphometric study. Synapse. 2005;57(1):47-55.

10. Dindot SV, Antalffy BA, Bhattacharjee MB, Beaudet AL. The Angelman syndrome ubiquitin ligase localizes to the synapse and nucleus, and maternal deficiency results in abnormal dendritic spine morphology. Human molecular genetics. 2008;17(1):111-118.

11. Chapleau CA, Calfa GD, Lane MC, et al. Dendritic spine pathologies in hippocampal pyramidal neurons from Rett syndrome brain and after expression of Rett-associated MECP2 mutations. Neurobiology of disease. 2009;35(2):219-233.

12. Landi S, Putignano E, Boggio EM, Giustetto M, Pizzorusso T, Ratto GM. The short-time structural plasticity of dendritic spines is altered in a model of Rett syndrome. Scientific reports. 2011;1:45.

13. Belichenko PV, Oldfors A, Hagberg B, Dahlstrom A. Rett syndrome: 3-D confocal microscopy of cortical pyramidal dendrites and afferents. Neuroreport. 1994;5(12):1509-1513.

14. Gao X, Deng P, Xu ZC, Chen J. Moderate traumatic brain injury causes acute dendritic and synaptic degeneration in the hippocampal dentate gyrus. PloS one. 2011;6(9):e24566.

15. Winston CN, Chellappa D, Wilkins T, et al. Controlled cortical impact results in an extensive loss of dendritic spines that is not mediated by injury-induced amyloid-beta accumulation. Journal of neurotrauma. 2013;30(23):1966-1972.

16. Scheff SW, DeKosky ST, Price DA. Quantitative assessment of cortical synaptic density in Alzheimer's disease. Neurobiology of aging. 1990;11(1):29-37.

17. DeKosky ST, Scheff SW. Synapse loss in frontal cortex biopsies in Alzheimer's disease: correlation with cognitive severity. Annals of neurology. 1990;27(5):457-464.

18. Masliah E, Terry RD, DeTeresa RM, Hansen LA. Immunohistochemical quantification of the synapse-related protein 
synaptophysin in Alzheimer disease. Neuroscience letters. 1989;103(2):234-239.

19. Masliah E, Terry R. The role of synaptic proteins in the pathogenesis of disorders of the central nervous system. Brain pathology. 1993;3(1):77-85.

20. Moolman DL, Vitolo OV, Vonsattel JP, Shelanski ML. Dendrite and dendritic spine alterations in Alzheimer models. Journal of neurocytology. 2004;33(3):377-387.

21. Schmitz AA, Govek EE, Bottner B, Van Aelst L. Rho GTPases: signaling, migration, and invasion. Experimental cell research. 2000;261(1):1-12.

22. Hanna S, El-Sibai M. Signaling networks of Rho GTPases in cell motility. Cellular signalling. 2013;25(10):1955-1961.

23. Spiering D, Hodgson L. Dynamics of the Rho-family small GTPases in actin regulation and motility. Cell adhesion \& migration. 2011;5(2):170-180.

24. Tashiro A, Minden A, Yuste R. Regulation of dendritic spine morphology by the rho family of small GTPases: antagonistic roles of Rac and Rho. Cerebral cortex. 2000;10(10):927-938.

25. Tashiro A, Yuste R. Regulation of dendritic spine motility and stability by Rac1 and Rho kinase: evidence for two forms of spine motility. Molecular and cellular neurosciences. 2004;26(3):429440.

26. Hall A. Rho GTPases and the actin cytoskeleton. Science. 1998;279(5350):509-514.

27. Hall A, Nobes CD. Rho GTPases: molecular switches that control the organization and dynamics of the actin cytoskeleton. Philosophical transactions of the Royal Society of London. Series B, Biological sciences. 2000;355(1399):965-970.

28. Pertz O. Spatio-temporal Rho GTPase signaling - where are we now? Journal of cell science. 2010;123(Pt 11):1841-1850.

29. Garcia-Mata R, Boulter E, Burridge K. The 'invisible hand': regulation of RHO GTPases by RHOGDIs. Nature reviews. Molecular cell biology. 2011;12(8):493-504.

30. Huesa G, Baltrons MA, Gomez-Ramos P, et al. Altered distribution of RhoA in Alzheimer's disease and AbetaPP overexpressing mice. Journal of Alzheimer's disease : JAD. 2010;19(1):37-56.

31. Petratos S, Li QX, George AJ, et al. The beta-amyloid protein of Alzheimer's disease increases neuronal CRMP-2 phosphorylation by a Rho-GTP mechanism. Brain : a journal of neurology. 2008;131(Pt 1):90-108.

32. Pozueta J, Lefort R, Ribe EM, Troy CM, Arancio O, Shelanski M. Caspase- 2 is required for dendritic spine and behavioural alterations in J20 APP transgenic mice. Nature communications. 2013;4:1939.

33. Saadipour K, Yang M, Lim Y, et al. Amyloid beta(1)(-)(4)(2) $(\operatorname{Abeta}(4)(2))$ up-regulates the expression of sortilin via the p75(NTR)/RhoA signaling pathway. Journal of neurochemistry. 2013;127(2):152-162.

34. Chacon PJ, Garcia-Mejias R, Rodriguez-Tebar A. Inhibition of RhoA GTPase and the subsequent activation of PTP1B protects cultured hippocampal neurons against amyloid beta toxicity. Molecular neurodegeneration. 2011;6(1):14.

35. Zhu X, Raina AK, Boux H, Simmons ZL, Takeda A, Smith MA. Activation of oncogenic pathways in degenerating neurons in Alzheimer disease. International journal of developmental neuroscience : the official journal of the International Society for Developmental Neuroscience. 2000;18(4-5):433-437.

36. Mendoza-Naranjo A, Gonzalez-Billault C, Maccioni RB. Abeta142 stimulates actin polymerization in hippocampal neurons through Rac1 and Cdc42 Rho GTPases. Journal of cell science. 2007;120(Pt 2):279-288.

37. Wang PL, Niidome T, Akaike A, Kihara T, Sugimoto H. Rac1 inhibition negatively regulates transcriptional activity of the amyloid precursor protein gene. Journal of neuroscience research. 2009;87(9):2105-2114.
38. Boo JH, Sohn JH, Kim JE, Song H, Mook-Jung I. Rac1 changes the substrate specificity of gamma-secretase between amyloid precursor protein and Notch1. Biochemical and biophysical research communications. 2008;372(4):913-917.

39. Zhou Y, Su Y, Li B, et al. Nonsteroidal anti-inflammatory drugs can lower amyloidogenic Abeta42 by inhibiting Rho. Science. 2003;302(5648):1215-1217.

40. Herskowitz JH, Feng Y, Mattheyses AL, et al. Pharmacologic inhibition of ROCK2 suppresses amyloid-beta production in an Alzheimer's disease mouse model. The Journal of neuroscience : the official journal of the Society for Neuroscience. 2013;33(49): 19086-19098.

41. Cerri C, Fabbri A, Vannini E, et al. Activation of Rho GTPases triggers structural remodeling and functional plasticity in the adult rat visual cortex. The Journal of neuroscience : the official journal of the Society for Neuroscience. 2011;31(42):15163-15172.

42. Borrelli S, Musilli M, Martino A, Diana G. Long-lasting efficacy of the cognitive enhancer cytotoxic necrotizing factor 1 . Neuropharmacology. 2013;64:74-80.

43. De Viti S, Martino A, Musilli M, Fiorentini C, Diana G. The Rho GTPase activating CNF1 improves associative working memory for object-in-place. Behavioural brain research. 2010;212(1):78-83.

44. Diana G, Valentini G, Travaglione S, et al. Enhancement of learning and memory after activation of cerebral Rho GTPases. Proceedings of the National Academy of Sciences of the United States of America. 2007;104(2):636-641.

45. Musilli M, Nicolia V, Borrelli S, Scarpa S, Diana G. Behavioral effects of Rho GTPase modulation in a model of Alzheimer's disease. Behavioural brain research. 2013;237:223-229.

46. De Filippis B, Fabbri A, Simone D, et al. Modulation of RhoGTPases improves the behavioral phenotype and reverses astrocytic deficits in a mouse model of Rett syndrome. Neuropsychopharmacology : official publication of the American College of Neuropsychopharmacology. 2012;37(5):1152-1163.

47. Mardilovich K, Olson MF, Baugh M. Targeting Rho GTPase signaling for cancer therapy. Future oncology. 2012;8(2):165-177.

48. Rex CS, Chen LY, Sharma A, et al. Different Rho GTPase-dependent signaling pathways initiate sequential steps in the consolidation of long-term potentiation. The Journal of cell biology. 2009;186(1): 85-97.

49. Murakoshi H, Wang H, Yasuda R. Local, persistent activation of Rho GTPases during plasticity of single dendritic spines. Nature. 2011;472(7341):100-104.

50. Alan JK, Lundquist EA. Mutationally activated Rho GTPases in cancer. Small GTPases. 2013;4(3):159-163.

51. van Galen EJ, Ramakers GJ. Rho proteins, mental retardation and the neurobiological basis of intelligence. Progress in brain research. 2005;147:295-317.

52. Ramakers GJ. Rho proteins and the cellular mechanisms of mental retardation. American journal of medical genetics. 2000;94(5):367371.

53. Raymond FL. X linked mental retardation: a clinical guide. Journal of medical genetics. 2006;43(3):193-200.

54. Chechlacz M, Gleeson JG. Is mental retardation a defect of synapse structure and function? Pediatric neurology. 2003;29(1):11-17.

55. Billuart P, Bienvenu T, Ronce N, et al. Oligophrenin-1 encodes a rhoGAP protein involved in X-linked mental retardation. Nature. 1998;392(6679):923-926.

56. Billuart P, Chelly J, Carrie A, et al. Determination of the gene structure of human oligophrenin-1 and identification of three novel polymorphisms by screening of DNA from 164 patients with non-specific X-linked mental retardation. Annales de genetique. 2000;43(1):5-9.

57. Fauchereau F, Herbrand U, Chafey P, et al. The RhoGAP activity of OPHN1, a new Factin-binding protein, is negatively controlled by 
its amino-terminal domain. Molecular and cellular neurosciences. 2003;23(4):574-586.

58. Govek EE, Newey SE, Akerman CJ, Cross JR, Van der Veken L, Van Aelst L. The Xlinked mental retardation protein oligophrenin-1 is required for dendritic spine morphogenesis. Nature neuroscience. 2004;7(4):364-372.

59. Kutsche K, Yntema H, Brandt A, et al. Mutations in ARHGEF6, encoding a guanine nucleotide exchange factor for Rho GTPases, in patients with X-linked mental retardation. Nature genetics. 2000;26(2):247-250.

60. Node-Langlois R, Muller D, Boda B. Sequential implication of the mental retardation proteins ARHGEF6 and PAK3 in spine morphogenesis. Journal of cell science. 2006;119(Pt 23):4986-4993.

61. Gubar OS, Houy S, Billuart P, et al. GTPase-activating protein oligophrenin 1 is a new partner of multifunctional adapter protein intersectin 1. Biopolymers and Cell. 2012;28(5):357-362.

62. Keating DJ, Chen C, Pritchard MA. Alzheimer's disease and endocytic dysfunction: clues from the Down syndrome-related proteins, DSCR1 and ITSN1. Ageing research reviews. 2006;5(4): 388-401.

63. Alam MR, Caldwell BD, Johnson RC, Darlington DN, Mains RE, Eipper BA. Novel proteins that interact with the COOH-terminal cytosolic routing determinants of an integral membrane peptideprocessing enzyme. The Journal of biological chemistry. 1996;271(45):28636-28640.

64. Ma XM, Kiraly DD, Gaier ED, et al. Kalirin-7 is required for synaptic structure and function. The Journal of neuroscience : the official journal of the Society for Neuroscience. 2008;28(47):1236812382.

65. Penzes P, Johnson RC, Alam MR, Kambampati V, Mains RE, Eipper BA. An isoform of kalirin, a brain-specific GDP/GTP exchange factor, is enriched in the postsynaptic density fraction. The Journal of biological chemistry. 2000;275(9):6395-6403.

66. Penzes P, Johnson RC, Sattler R, et al. The neuronal Rho-GEF Kalirin-7 interacts with PDZ domain-containing proteins and regulates dendritic morphogenesis. Neuron. 2001;29(1):229-242.

67. Xie Z, Srivastava DP, Photowala H, et al. Kalirin-7 controls activitydependent structural and functional plasticity of dendritic spines. Neuron. 2007;56(4):640-656.

68. Ma XM, Huang JP, Kim EJ, et al. Kalirin-7, an important component of excitatory synapses, is regulated by estradiol in hippocampal neurons. Hippocampus. 2011;21(6):661-677.

69. McPherson CE, Eipper BA, Mains RE. Genomic organization and differential expression of Kalirin isoforms. Gene. 2002;284(1-2): $41-51$.

70. Johnson RC, Penzes P, Eipper BA, Mains RE. Isoforms of kalirin, a neuronal $\mathrm{Dbl}$ family member, generated through use of different 5 'and 3'-ends along with an internal translational initiation site. The Journal of biological chemistry. 2000;275(25):19324-19333.

71. McPherson CE, Eipper BA, Mains RE. Kalirin expression is regulated by multiple promoters. Journal of molecular neuroscience : MN. 2004;22(1-2):51-62.

72. Remmers C, Sweet RA, Penzes P. Abnormal kalirin signaling in neuropsychiatric disorders. Brain research bulletin. 2014;103:2938.

73. Muller D, Buchs PA, Stoppini L. Time course of synaptic development in hippocampal organotypic cultures. Brain research. Developmental brain research. 1993;71(1):93-100.

74. Ma XM, Huang J, Wang Y, Eipper BA, Mains RE. Kalirin, a multifunctional Rho guanine nucleotide exchange factor, is necessary for maintenance of hippocampal pyramidal neuron dendrites and dendritic spines. The Journal of neuroscience : the official journal of the Society for Neuroscience. 2003;23(33):10593-10603.

75. Youn H, Jeoung M, Koo Y, et al. Kalirin is under-expressed in Alzheimer's disease hippocampus. Journal of Alzheimer's disease : JAD. 2007;11(3):385-397.
76. Youn H, Ji I, Ji HP, Markesbery WR, Ji TH. Under-expression of Kalirin-7 Increases iNOS activity in cultured cells and correlates to elevated iNOS activity in Alzheimer's disease hippocampus. Journal of Alzheimer's disease : JAD. 2007;12(3):271-281.

77. Murray PS, Kirkwood CM, Gray MC, et al. beta-Amyloid 42/40 ratio and kalirin expression in Alzheimer disease with psychosis. Neurobiology of aging. 2012;33(12):2807-2816.

78. Ratovitski EA, Alam MR, Quick RA, et al. Kalirin inhibition of inducible nitric-oxide synthase. The Journal of biological chemistry. 1999;274(2):993-999.

79. Luth HJ, Munch G, Arendt T. Aberrant expression of NOS isoforms in Alzheimer's disease is structurally related to nitrotyrosine formation. Brain research. 2002;953(1-2):135-143.

80. Aliev G, Palacios HH, Lipsitt AE, et al. Nitric oxide as an initiator of brain lesions during the development of Alzheimer disease. Neurotoxicity research. 2009;16(3):293-305.

81. Namekata K, Kimura A, Kawamura K, Harada C, Harada T. Dock GEFs and their therapeutic potential: Neuroprotection and axon regeneration. Progress in retinal and eye research. 2014.

82. Miyamoto Y, Yamauchi J. Cellular signaling of Dock family proteins in neural function. Cellular signalling. 2010;22(2):175-182.

83. Kashiwa A, Yoshida H, Lee S, et al. Isolation and characterization of novel presenilin binding protein. Journal of neurochemistry. 2000;75(1):109-116.

84. Namekata K, Enokido Y, Iwasawa K, Kimura H. MOCA induces membrane spreading by activating Rac1. The Journal of biological chemistry. 2004;279(14):14331-14337.

85. Chen Q, Yoshida H, Schubert D, Maher P, Mallory M, Masliah E. Presenilin binding protein is associated with neurofibrillary alterations in Alzheimer's disease and stimulates tau phosphorylation. The American journal of pathology. 2001;159(5):1597-1602.

86. Chen Q, Kimura H, Schubert D. A novel mechanism for the regulation of amyloid precursor protein metabolism. The Journal of cell biology. 2002;158(1):79-89.

87. Pasquale EB. Eph-ephrin bidirectional signaling in physiology and disease. Cell. 2008;133(1):38-52.

88. Chen Y, Fu AK, Ip NY. Eph receptors at synapses: implications in neurodegenerative diseases. Cellular signalling. 2012;24(3):606611.

89. Kullander K, Klein R. Mechanisms and functions of Eph and ephrin signalling. Nature reviews. Molecular cell biology. 2002;3(7):475486.

90. Henkemeyer M, Itkis OS, Ngo M, Hickmott PW, Ethell IM. Multiple EphB receptor tyrosine kinases shape dendritic spines in the hippocampus. The Journal of cell biology. 2003;163(6):13131326.

91. Fu WY, Chen Y, Sahin M, et al. Cdk5 regulates EphA4-mediated dendritic spine retraction through an ephexin1-dependent mechanism. Nature neuroscience. 2007;10(1):67-76.

92. Murai KK, Nguyen LN, Irie F, Yamaguchi Y, Pasquale EB. Control of hippocampal dendritic spine morphology through ephrin-A3/ EphA4 signaling. Nature neuroscience. 2003;6(2):153-160.

93. Grunwald IC, Korte M, Adelmann G, et al. Hippocampal plasticity requires postsynaptic ephrinBs. Nature neuroscience. 2004;7(1): 33-40.

94. Torres R, Firestein BL, Dong H, et al. PDZ proteins bind, cluster, and synaptically colocalize with Eph receptors and their ephrin ligands. Neuron. 1998;21(6):1453-1463.

95. Bruckner K, Pablo Labrador J, Scheiffele P, Herb A, Seeburg PH, Klein R. EphrinB ligands recruit GRIP family PDZ adaptor proteins into raft membrane microdomains. Neuron. 1999;22(3):511-524.

96. Dalva MB, Takasu MA, Lin MZ, et al. EphB receptors interact with NMDA receptors and regulate excitatory synapse formation. Cell. 2000;103(6):945-956. 
97. Henderson JT, Georgiou J, Jia Z, et al. The receptor tyrosine kinase EphB2 regulates NMDA-dependent synaptic function. Neuron. 2001;32(6):1041-1056.

98. Takasu MA, Dalva MB, Zigmond RE, Greenberg ME. Modulation of NMDA receptordependent calcium influx and gene expression through EphB receptors. Science. 2002;295(5554):491-495.

99. Margolis SS, Salogiannis J, Lipton DM, et al. EphB-mediated degradation of the RhoA GEF Ephexin5 relieves a developmental brake on excitatory synapse formation. Cell. 2010;143(3):442-455.

100. Simón AM, de Maturana RL, Ricobaraza A, et al. Early changes in hippocampal Eph receptors precede the onset of memory decline in mouse models of Alzheimer\&apos;s disease. Journal of Alzheimer\&apos;s disease : JAD. 2009;17(4):773-786.

101. Cisse M, Halabisky B, Harris J, et al. Reversing EphB2 depletion rescues cognitive functions in Alzheimer model. Nature. 2011;469(7328):47-52.

102. Xu J, Litterst C, Georgakopoulos A, Zaganas I, Robakis NK. Peptide EphB2/CTF2 generated by the gamma-secretase processing of EphB2 receptor promotes tyrosine phosphorylation and cell surface localization of N-methyl-D-aspartate receptors. The Journal of biological chemistry. 2009;284(40):27220-27228.

103. Irie F, Yamaguchi Y. EphB receptors regulate dendritic spine development via intersectin, Cdc42 and N-WASP. Nature neuroscience. 2002;5(11):1117-1118.

104. Murata Y, Constantine-Paton M. Postsynaptic density scaffold SAP102 regulates cortical synapse development through EphB and PAK signaling pathway. The Journal of neuroscience : the official journal of the Society for Neuroscience. 2013;33(11):5040-5052.

105. Fu AK, Hung KW, Fu WY, et al. APC(Cdh1) mediates EphA4dependent downregulation of AMPA receptors in homeostatic plasticity. Nature neuroscience. 2011;14(2):181-189.

106. Carmona MA, Murai KK, Wang L, Roberts AJ, Pasquale EB. Glial ephrin-A3 regulates hippocampal dendritic spine morphology and glutamate transport. Proceedings of the National Academy of Sciences of the United States of America. 2009;106(30):1252412529.
107. O'Leary DD, Wilkinson DG. Eph receptors and ephrins in neural development. Current opinion in neurobiology. 1999;9(1):65-73.

108. Williams C, Mehrian Shai R, Wu Y, et al. Transcriptome analysis of synaptoneurosomes identifies neuroplasticity genes overexpressed in incipient Alzheimer's disease. PloS one. 2009;4(3):e4936.

109. Rosenberger A, Rozemuller A, van der Flier WM, Scheltens P, van der Vies SM, Hoozemans J. Altered distribution of the EphA4 kinase in hippocampal brain tissue of patients with Alzheimer inverted question marks disease correlates with pathology. Acta neuropathologica communications. 2014;2(1):79.

110. Vargas LM, Leal N, Estrada LD, et al. EphA4 activation of c-Abl mediates synaptic loss and LTP blockade caused by amyloid-beta oligomers. PloS one. 2014;9(3):e92309.

111. Hemming ML, Elias JE, Gygi SP, Selkoe DJ. Identification of betasecretase (BACE1) substrates using quantitative proteomics. PloS one. 2009;4(12):e8477.

112. Inoue $\mathrm{E}$, Deguchi-Tawarada $\mathrm{M}$, Togawa $\mathrm{A}$, et al. Synaptic activity prompts gammasecretase-mediated cleavage of EphA4 and dendritic spine formation. The Journal of cell biology. 2009;185(3):551564.

113. Shi L, Fu WY, Hung KW, et al. Alpha2-chimaerin interacts with EphA4 and regulates EphA4-dependent growth cone collapse. Proceedings of the National Academy of Sciences of the United States of America. 2007;104(41):16347-16352.

114. Noberini R, Koolpe M, Peddibhotla S, et al. Small molecules can selectively inhibit ephrin binding to the EphA4 and EphA2 receptors. The Journal of biological chemistry. 2008;283(43):2946129472.

115. Fu AK, Hung KW, Huang H, et al. Blockade of EphA4 signaling ameliorates hippocampal synaptic dysfunctions in mouse models of Alzheimer's disease. Proceedings of the National Academy of Sciences of the United States of America. 2014;111(27):99599964.

116. Parri M, Chiarugi P. Rac and Rho GTPases in cancer cell motility control. Cell communication and signaling : CCS. 2010;8:23. 